\title{
A $p$-ADIC REGULATOR PROBLEM IN ALGEBRAIC $K$-THEORY AND GROUP COHOMOLOGY
}

\author{
BY J. B. WAGONER ${ }^{1}$
}

Let $O$ be the ring of integers in a number field $F$. Let $\mathfrak{p} \subset O$ be a prime ideal and $O_{\mathfrak{p}}=\varliminf O / \mathfrak{p}^{s}$ be the $\mathfrak{p}$-adic completion of $O$. Let

$$
\begin{aligned}
& \hat{K}_{n}(0)=K_{n}(0) \quad \bmod \text { torsion, } \\
& \hat{K}_{n}^{c}\left(O_{\mathfrak{p}}\right)=K_{n}^{c}\left(O_{\mathfrak{p}}\right) \bmod \text { torsion, }
\end{aligned}
$$

where $K_{n}(0)$ is the algebraic $K$-theory of Quillen [Q] and

$$
K_{n}^{c}\left(O_{\mathfrak{p}}\right)=\varliminf_{n} K_{n}\left(O / \mathfrak{p}^{s}\right)
$$

is the "continuous" or " $p$-adic" algebraic $K$-theory of $O_{\mathfrak{p}}$ studied in [W1] by Milgram and the author. Results of [B] and [W1] suggested asking whether

$$
\Phi_{\mathfrak{p}}: \hat{K}_{n}(0) \rightarrow \hat{K}_{n}^{c}\left(O_{\mathfrak{p}}\right)
$$

or

$$
\Phi: \hat{K}_{n}(0) \rightarrow \bigoplus_{\mathfrak{p} \mid p} \hat{K}_{n}^{c}\left(O_{\mathfrak{p}}\right)
$$

is injective, where $p$ is a fixed rational prime and $n>1$ is odd. Observe that each $\Phi_{\mathfrak{p}}$ is clearly injective for $n=1$, because $K_{1}(0)=0^{*}$ and $K_{1}^{c}\left(O_{\mathfrak{p}}\right)=0_{\mathfrak{p}}^{*}$. A much harder problem is whether $\Phi \otimes Z_{p}$ is injective. For $n=1$ and $F$ totally real abelian, injectively of $\Phi \otimes \mathbf{Z}_{p}$ on the subgroup of $0^{*}$ consisting of those elements congruent to $1 \bmod \mathfrak{p}$ for each $\mathfrak{p} \mid p$ is equivalent to nonvanishing of the $p$-adic regulator $[\mathbf{B r}, \mathbf{C}]$. As an example of (1) let $F$ be quadratic imaginary. Then is

$$
\Phi_{\mathfrak{p}}: \mathbf{Z} \cong \hat{K}_{3}(0) \rightarrow \hat{K}_{3}^{c}\left(O_{\mathfrak{p}}\right) \cong \mathbf{Z}_{p}
$$

injective when $p=\operatorname{char}(O / \mathfrak{p})$ is unramified with $O_{\mathfrak{p}} \cong \mathbf{Z}_{p}$ ? J.-P. Serre asked an equivalent cohomological version of (1) and (2) prior to the circa $1975 \mathrm{~K}$ theory formulation. For special case (3) injectivity is equivalent to showing $\Phi_{\mathfrak{p}} \otimes Q_{p}$ is an isomorphism, which in turn amounts to showing

$$
Q_{p} \cong H_{c}^{3}\left(\mathrm{SL}_{n}\left(O_{\mathfrak{p}}\right) ; Q_{p}\right) \rightarrow H^{3}\left(\mathrm{SL}_{n}(O) ; Q_{p}\right) \cong Q_{p}
$$

is an isomorphism for $n$ large. $H_{c}^{3}$ denotes the continuous cohomology of the $p$-adic group $\mathrm{SL}_{n}\left(O_{\mathfrak{p}}\right)$ and $H^{3}$ is the Eilenberg-Mac Lane cohomology of the discrete group $\mathrm{SL}_{n}(0)$. Compare $[\mathbf{L}]$. Numerous examples of (4)

Received by the editors May 12, 1983.

1980 Mathematics Subject Classification. Primary 18F25, 12B30; Secondary 12B22, 57T10.

${ }^{1}$ Partially supported by NSF Grant MCS80-0258 and DOE Grant DE-AT03-762F0034 Project Agreement DEAS03-79ER10358. 
result from nonvanishing of the Gross-Coleman $Q_{p}$-regulator as formulated in [Co]. This regulator connects the $p$-adic dilogarithm and the $L$-function values $L_{p}\left(2, \chi \omega^{-1}\right)$.

There is the companion $\mathbf{Z}_{p}$-regulator question to (3): namely, determine the index $R_{p}$ of

$$
\Phi_{\mathfrak{p}} \otimes \mathbf{Z}_{p}: \mathbf{Z}_{p} \cong \hat{K}_{3}(0) \otimes \mathbf{Z}_{p} \rightarrow \hat{K}_{3}^{c}\left(O_{\mathfrak{p}}\right) \cong \mathbf{Z}_{p}
$$

For $F=Q(\sqrt{-3})=Q(\mu)$, where $\mu^{3}=1$, we give examples for which $R_{p}=$ 1, i.e., for which $\Phi_{\mathfrak{p}} \otimes \mathbf{Z}_{p}$ is an isomorphism. This is done with the aid of a homomorphism $\mathrm{Ch}_{\mathfrak{p}}: K_{3}(\mathrm{O}) \rightarrow \mathrm{Z} / p$ constructed by elementary methods, and the values of $\mathrm{Ch}_{\mathfrak{p}}$ turn out to be related experimentally to the values $L_{p}\left(2, \chi \omega^{-1}\right) \bmod p$, where $\chi$ is the Dirichlet character of conductor 3 and $\omega$ is the Teichmüller character on $\mathbf{Z}_{p}^{*}$. The details are in [W2].

Aisbett $[\mathbf{A}]$ has shown $K_{3}\left(\mathbf{Z} / p^{n}\right)=\mathbf{Z} / p^{2(n-1)} \oplus \mathbf{Z} / p^{2}-1$ for $p>2$. To test for examples where (5) is an isomorphism it is sufficient to

(a) find an explicit element $B \in K_{3}(0)$,

(b) find an explicit formula for a homomorphism

$$
\mathrm{Ch}_{\mathfrak{p}}: K_{3}(0) \rightarrow K_{3}^{c}\left(O_{\mathfrak{p}}\right) \rightarrow K_{3}\left(\mathbf{Z} / p^{2}\right) \rightarrow \mathbf{Z} / p
$$

(c) determine $\operatorname{Ch}_{\mathfrak{p}}(B) \neq 0$ in various cases by machine computation.

In the case $F=Q(\sqrt{-3})$, Tate has shown $K_{2}(0)=0$. Hence $K_{3}(0)$ $=H_{3}\left(\mathrm{St}_{n}(0)\right)=H_{3}\left(E_{n}(0)\right)$ for $n$ large enough, where $E_{n}(0)$ is the group of $n \times n$ elementary matrices. The class $B \in H_{3}\left(E_{n}(O)\right)$ is represented as an explicit sum of 30 simplices in the bar resolution of $E_{3}(O)$, and the construction of $B$ makes use of Riley's hyperbolic representation of the fundamental group of the complement of the figure eight knot $[\mathbf{R}, \mathbf{M}]$. As a cohomology class, the homomorphism $\mathrm{Ch}_{\mathfrak{p}}: K_{3}(\mathrm{O}) \rightarrow \mathrm{Z} / p$ comes from the diagram

$$
\begin{aligned}
& K_{3}(0) \rightarrow K_{3}^{c}\left(O_{\mathfrak{p}}\right) \rightarrow K_{3}\left(0 / \mathfrak{p}^{2}\right) \cong K_{3}\left(\mathbf{Z} / p^{2}\right) \\
& \begin{array}{cccc}
\downarrow & \downarrow & \downarrow & \downarrow \\
H_{3}(E(O)) & \rightarrow & H_{3}\left(E\left(O / \mathfrak{p}^{2}\right)\right) \cong H_{3}\left(E\left(\mathbf{Z} / p^{2}\right)\right) \underset{\mathrm{ch}}{\rightarrow \mathbf{Z}} / p
\end{array}
\end{aligned}
$$

The explicit formula for the $E\left(\mathbf{Z} / p^{2}\right)$ invariant cocycle ch on a three simplex $g[a|b| c]$ in the bar resolution arises from examination of the standard cohomology class $\Delta \in H^{2}\left(\mathrm{GL}_{n}(\mathrm{Z} / p) ; M_{n}(\mathrm{Z} / p)\right)$ of the extension

$$
0 \rightarrow M_{n}(\mathbf{Z} / p) \rightarrow \mathrm{GL}_{n}\left(\mathbf{Z} / p^{2}\right) \rightarrow \mathrm{GL}_{n}(\mathbf{Z} / p) \rightarrow 1
$$

The class ch is a special case of a class constructed in $H^{3}\left(\mathrm{GL}_{n}\left(A / I^{2}\right) ; I^{2} / I^{3}\right)$ when $A$ is semilocal with radical $I$ such that $A / I$ is finite.

Let $F=Q(\sqrt{-3})$ and recall [Coh] that a rational prime $p>3$ is split iff -3 is a quadratic residue $\bmod p$. In this case solve $x^{2} \equiv-3 \bmod p$. Then $\langle p\rangle=\mathfrak{p} \overline{\mathfrak{p}}$, where $\mathfrak{p}=\langle p, x+\sqrt{-3}\rangle, \overline{\mathfrak{p}}=\langle p,-x+\sqrt{-3}\rangle$, and $\mathcal{O}_{\mathfrak{p}} \cong \mathcal{O}_{\mathfrak{p}} \cong \mathbf{Z}_{p}$. 
THEOREM. In the following cases $\Phi_{\mathfrak{p}} \otimes \mathbf{Z}_{p}$ is an isomorphism because $\mathrm{Ch}_{\mathfrak{p}}(B) \neq 0$ :

$\begin{array}{rrc}p & x & \operatorname{Ch}_{\mathfrak{p}}(B) \text { for } \mathfrak{p}=\langle p, x+\sqrt{-3}\rangle \\ 7 & 2 & 1 \\ 13 & 6 & 2 \\ 19 & 4 & 3 \\ 31 & 11 & 22 \\ 37 & 16 & 2 \\ 43 & 13 & 9 \\ 61 & 27 & 27 \\ 67 & 8 & 33 \\ 73 & 17 & 19\end{array}$

In general we have $\mathrm{Ch}_{\mathfrak{p}}(B)=-\mathrm{Ch}_{\mathfrak{p}}(B)$ so there are nine more cases where $\mathrm{Ch}_{\mathfrak{p}} \neq 0$. The above examples were the only one computed. To make the computation for $\mathrm{Ch}_{\mathfrak{p}}$ we use the isomorphism $i_{\mathfrak{p}}: 0 / \mathfrak{p}^{2} \rightarrow \mathrm{Z} / p^{2}$ arising from

$$
a+b \nu \rightarrow a+b\left(\left(3+2 x-x^{2}\right) / 4 x\right) \bmod p^{2},
$$

where $\nu=(1+\sqrt{-3}) / 2$ and $a, b \in \mathbf{Z}$.

In [Co] Coleman uses the $p$-adic dilogarithm to define a homomorphism $D_{p}^{*}: K_{3}\left(C_{p}\right) \rightarrow C_{p}$, where $C_{p}$ is a completion of the algebraic closure of $Q_{p}$. When $O$ is the integers in the number field of $m$ th roots of unity, he proves a regulator formula for $K_{3}(0)$ involving $D_{p}^{*}$ and $L_{p}\left(2, \chi \omega^{-1}\right)$, where $\chi$ has conductor $m$ and $\omega$ is the Teichmüller character on $\mathbf{Z}_{p}^{*}$. In the case $m=3$, Theorem 8.1 of [Co] suggests, after simplification, that we should have

$$
L_{p}\left(2, \chi \omega^{-1}\right) \equiv-r_{B} i_{\mathfrak{p}}(x) \mathrm{Ch}_{\mathfrak{p}}(B) \bmod p
$$

for some rational number $r_{B}$ depending only on $B$ and having denominator prime to $p$. The factor $r_{B}$ occurs because we only know $B \in \hat{K}_{3}(0)$ is some integer multiple of the generator. Machine computation verifies (6) holds for $r_{B}=1 / 18$ in all cases of $\mathfrak{p}=\langle p, \pm x+\sqrt{-3}\rangle$ considered above.

It is a pleasure to acknowledge the assistance of R. Fateman, K. Sklower, and others of the Berkeley Computer Science Department as well as O. Lanford and J. Wolf in the Mathematics Department. Their help in introducing the author to Berkeley's computer facilities was invaluable in expediting this project in a timely fashion. We would also like to thank K. Ribet for stimulating discussion on $L$-functions.

\section{REFERENCES}

A. J. Aisbett, $O n K_{3}$ and $K_{4}$ of integers $\bmod n$, Bull. Amer. Math. Soc. (N.S.) 6 (1982), 417-420.

B. A. Borel, Stable real cohomology of arithmetic groups, Ann. Sci. École Norm. Sup. (4) 7 (1974), 253-272.

Br. A. Brumer, On the units of algebraic number fields, Mathematika 14 (1967), 121-124.

C. J. Coates, On the values of the p-adic zeta functions at the odd positive integers, Notes, Univ. de Paris, Orsay. 
Co. R. Coleman, Dilogarithms, regulators, and p-adic L-functions, Invent. Math. 69 (1982), 171-208.

Coh. H. Cohn, A second course in number theory, Wiley, New York, 1962.

L. M. Lazard, Groupes analytiques p-adic, Inst. Hautes Études Sci. Publ. Math. No. 26 (1965).

M. J. Milnor, Hyperbolic geometry: The first 150 years, Bull. Amer. Math. Soc. (N.S.) 6 (1982), 9-24.

Q. D. Quillen, Higher algebraic K-theory. I, Lecture Notes in Math., vol. 341, SpringerVerlag, pp. 85-147.

R. R. Riley, A quadratic parabolic group, Math. Proc. Cambridge Philos. 77 (1945), 281288.

W1. J. Wagoner, Continuous cohomology and $p$-adic $K$-theory, Lecture Notes in Math., vol. 551, Springer-Verlag, pp. 241-248.

W2. 1983.

Department of Mathematics, University of California, Berkeley, CaliFORNIA 94720 